\title{
Comparative Activity and Specificity of Antisense Oligodeoxynucleotides and Small Interfering RNA in an in vitro Ewing Sarcoma Model
}

\author{
Marie Villemeur ${ }^{1}$, Ali Tamaddon ${ }^{1,2}$, Jean-Rémi Bertrand ${ }^{1}$ and Claude Malvy*,1 \\ ${ }^{1}$ Laboratoire de Vectorologie et Transfert de Gènes, Institut Gustave Roussy, UMR CNRS 8121, 39, rue Camille \\ Desmoulins, 94805 Villejuif Cedex, France \\ ${ }^{2}$ Department of Pharmaceutics, School of Pharmacy, Shiraz University of Medical Sciences, P.O. Box 14155-6153, \\ Tehran, Iran
}

\begin{abstract}
The EWS-Fli1 fusion gene, resulting from a t(11;22) translocation, plays a key role in the Ewing's sarcoma pathogenesis. In the past, a $25 \mathrm{mer}$ phosphorothioate antisense oligodeoxynucleotide, a structured 30mer phosphorothioate/phosphodiester antisense oligodeoxynucleotide, and an antisense siRNA, delivered either free, by vectors or intracellularly expressed, were found potent in various in vitro and in vivo Ewing sarcoma models. Because of differences among the models used in the literature, the comparison of various antisense agents with each other is difficult. Therefore, we aimed to evaluate these three antisense agents in NIH/3T3 fibroblasts which stably express the human EWS-Fli1 oncogene as an in vitro model of Ewing sarcoma. Fours parameters were considered including oncogene EWS-Fli1 and EWS mRNA expression, cellular proliferation, and actin cytoskeleton organization. They illustrate the antisense efficacy, the specificity and the phenotypic reversion for the last two ones, respectively. We showed that the structured 30mer phosphorothioate/phosphodiester antisense oligodeoxynucleotide and antisense siRNA represent the best choice for clinical trials. Nevertheless, the antisense ODN is more specific than the siRNA and represents the most efficient antisense agent. Its activity may be improved after the selection of an appropriate delivery vector which is able to increase cell penetration and to protect it from nucleases degradation.
\end{abstract}

\section{INTRODUCTION}

The Ewing sarcoma family of tumours (ESFT) is the second most common malignant neoplasm of bone among children and adolescents. ESFT comprises tumours which are characterised by specific translocations involving the EWS gene and one of several members of the ETS family of transcription factors. This family includes a great number of oncogenic transcription factors involved in the regulation of tumor and developmental processes. In $90-95 \%$ of the cases, the translocation $\mathrm{t}(11 ; 22)(\mathrm{q} 24 ; 12)$ creates the EWS-Fli1 fusion gene. This results in the expression of a chimerical oncoprotein consisting of the N-terminal domain of ews and the DNA-binding domain of flil. Depending on the locations of the EWS and Fli1 genomic breakpoints, several types of EWS-Fli1 fusion gene exist. The fusion of EWS exon 7 to Fli1 exon 6 (type 1) is the most common (60\% of cases). The ews-flil oncoprotein acts as an aberrant transcription factor. Because it modulates the expression of various target genes, ews-flil is considered as the main cause of Ewing sarcoma [1-3].

A new therapeutic strategy against Ewing sarcoma would be to block specifically the expression of the ews-flil oncoprotein. Therefore, we focus on the very powerful antisense tools which specifically inhibit gene expression at RNA level and then block the encoded protein synthesis: short frag-

\footnotetext{
*Address correspondence to this author at the Laboratoire de Vectorologie et Transfert de Gènes, Institut Gustave Roussy, UMR CNRS 8121, 39, rue Camille Desmoulins, 94805 Villejuif Cedex, France; Tel: +33 142115 402; Fax: +33 142115 245; E-mail: cmalvy@igr.fr
}

ments of single-stranded DNA called antisense oligodeoxynucleotides (AS ODNs) and small interfering RNAs (siRNAs). The use of such technology was early studied on Ewing sarcoma models [4-16]. We will consider either AS ODNs or siRNAs all directed against the type 1 junction breakpoint of human EWS-Fli1 mRNA. Tanaka et al. were the first to use AS ODN with phosphorothioate (PS) linkages. In the micro molar range and without transfection agent, a 25mer PS AS ODN was enough efficient to inhibit the growth of SK-N-MC cells in culture [6]. In a mouse xenograft model of human EWS-Fli1 transformed NIH/3T3 cells, Lambert et al. enclosed the same 25mer PS AS ODN in biocompatible polymeric nanocapsules and observed $70 \%$ inhibition of tumour growth after intratumoural injection at a cumulative dose of $5 \mathrm{mg} / \mathrm{kg}$ [7]. Maksimenko et al. developed a structured 30mer AS ODN composed by 22 phosphodiester (PO) nucleotides and 8 PS nucleotides. Its internal structure allows protection against nuclease degradation with the advantage of a low number of thioate groups. The excess of thioate groups is well known to induce nonspecific effects and may be responsible for toxicity. When the structured 30mer PS/PO AS ODN was delivered intratumourally by nanocapsules or by chitosan nanospheres (cumulative dose of 4 and $5 \mathrm{mg} / \mathrm{kg}$ respectively), they obtained $82 \%$ and $66 \%$ inhibition of EWS-Fli1 tumour growth in nude mice [10,11]. At $200 \mathrm{nM}$, the structured 30mer PS/PO AS ODN bound to chitosan nanospheres or to cationic lipids inhibited both EWS-Fli1 mRNA and protein expression by $50 \%$, in NIH/3T3 permanently expressing human EWS-Fli1 cells culture. Only with cationic lipids, the authors observed also a 50\% inhibition of EWS-Fli1 NIH/3T3 cells growth in 
vitro. On the other hand, the growth inhibition of xenografted EWS-Fli1 NIH/3T3 tumours was noted after intratumoural or intravenous injection of structured $30 \mathrm{mer}$ PS/PO AS ODN bound to chitosan nanospheres [14]. Finally, according to Dohjima et al., one AS siRNA intracellularly expressed involved $81 \%$ reduction of EWS-Fli1 mRNA expression and 50\% Ewing sarcoma cell line TC135 growth inhibition [9]. In a mouse xenograft model of human EWSFli1 transformed NIH/3T3 cells, this AS siRNA enclosed in biocompatible polymeric nanocapsules were found to trigger a dose-dependant inhibition of tumour growth after intratumoural injection $(43 \%$ and $80 \%$ inhibition at $0.8 \mathrm{mg} / \mathrm{kg}$ and $1.11 \mathrm{mg} / \mathrm{kg}$, respectively). These results were linked to an inhibition of EWS-Fli1 gene expression [16].

Each of these three-presented antisense agents (25mer PS AS ODN, structured 30mer PS/PO AS ODN, AS siRNA) constitutes prospects of an efficient Ewing sarcoma treatment. Unfortunately, the experimental conditions are extremely different from a study to another. So, it is difficult to compare the antisense agents with each other. In order to select the most efficient, we compared them in the same cell culture conditions after vectorisation by cationic lipids. First, using an in vitro model of Ewing sarcoma (NIH/3T3 stably expressing human EWS-Fli1 oncogene), we determined their antisense efficacy via their ability to inhibit the oncogene expression. Second, as EWS-Fli1 fusion gene consists of the first seven exons of EWS joined to Fli1 from its exon 6, we controlled the specificity of antisense agents by checking if the EWS gene expression is modified in human EWS-Fli1 transformed murine fibroblasts and parental NIH/3T3 cells. Indeed, EWS has been shown to act as an adaptor molecule linking gene transcription and mRNA processing by interacting with RNA polymerase II and the splicing factors. Consequently, the modification of its physiological expression in normal cells may have undesirable effect [17-19]. Third, the human EWS-Fli1 expression into NIH/3T3 cells increases their growing rate in comparison with the untransformed $\mathrm{NIH} / 3 \mathrm{~T} 3$ cells. We evaluated the effect of these three antisense agents on the cell proliferation. Fourth, common features including disruption of actin filaments are noticed after transformation of cells by various oncogenes. These changes in microfilament structure are highly related to both anchorage-independent growth and cellular tumourigenicity, suggesting fundamental roles for actin filaments in oncogenic transformation [20]. In this way, NIH/3T3 fibroblasts feature a highly organised actin filament network whereas tumour EWS-Fli1 NIH/3T3 cells exhibit a complete disruption of their actin cytoskeleton [21]. So, we evaluated the different antisense agents according to their ability to restore actin fibres network.

The aim of this work was to compare the efficiency of three different antisense agents described in the literature on their ability to block specifically EWS-Fli1 expression and the phenotypes associated to the cellular transformation. Finally, we are capable of selecting a good candidate to design a new therapy for patients with Ewing sarcoma.

\section{MATERIALS AND METHODS}

\section{Cell Lines}

As a model of Ewing sarcoma, we used the fibroblastic NIH/3T3 cell line stably transfected with the human EWSFli1 junction oncogene which was a generous gift from Dr J. Ghysdael (Institut Curie; Orsay, France). The cells were grown in DMEM containing glucose $(4500 \mathrm{mg} / \mathrm{L})$ and glutamax I (GIBCO; Cergy-Pontoise, France), supplemented by penicillin $(100 \mathrm{U} / \mathrm{mL}$; GIBCO), streptomycin $(100 \mu \mathrm{g} / \mathrm{mL}$; GIBCO), $10 \%$ heat-inactivated new born bovine serum (GIBCO), and puromycin $(2.5 \mu \mathrm{g} / \mathrm{mL}$; Sigma; Lyon, France) at $37{ }^{\circ} \mathrm{C}$ in a $5 \% \quad \mathrm{CO}_{2}$ water-saturated atmosphere. As a negative control, NIH/3T3 cell line was maintained under the same conditions without puromycin. The parental NIH/3T3 fibroblasts only express the EWS gene whereas the tumour EWS-Fli1 NIH/3T3 cells express both the EWS and the EWS-Fli1 genes.

\section{Antisense Oligodeoxynucleotides (AS ODNs) and Small Interfering RNA (siRNAs)}

The oligodeoxynucleotides, Fig. (1), directed against the breakpoint of the type 1 human EWS-Fli1 fusion gene were synthesized and purified by Eurogentec (Seraing, Belgium). The first one is a $25 \mathrm{mer}$ fully constituted by phosphorothioate linkages (which are noted by*) with the following sequence: $5{ }^{\prime}-\mathrm{G}^{*} \mathrm{~A} * \mathrm{C} * \mathrm{~T}^{*} \mathrm{G} * \mathrm{~A} * \mathrm{G} * \mathrm{~T} * \mathrm{C} * \mathrm{~A} * \mathrm{~T}^{*} \mathrm{~A} * \mathrm{~A} * \mathrm{G} *$ $A^{*} A^{*} \mathrm{G}^{*} \mathrm{G}^{*} \mathrm{G}^{*} \mathrm{~T} * \mathrm{~T}^{*} \mathrm{C}^{*} \mathrm{~T} * \mathrm{G}^{*} \mathrm{C}-3^{\prime}$ (25mer PS AS ODN) [6]. The second one presents a stem loop structure in charge of protecting them from nuclease degradation with a low number of chemically modified bases to decrease the unspecific effects. This ODN contains a 22 phosphodiesters stem and a 8 phosphorothioates loop: 5'-GTAGCGAAGGGT*T*C*T* $\mathrm{G}^{*} \mathrm{C}^{*} \mathrm{~T}^{*} \mathrm{G}^{*} \mathrm{CCCGTAGCTGC-3}$ (structured 30mer PS/PO AS ODN; application for a patent Bioalliance Pharma S.A., CNRS, IGR, No 99/02921, 09/03/1999) [11]. As the two ODNs are directed against the junction breakpoint of EWS-

Type 1 Breakpoint junction

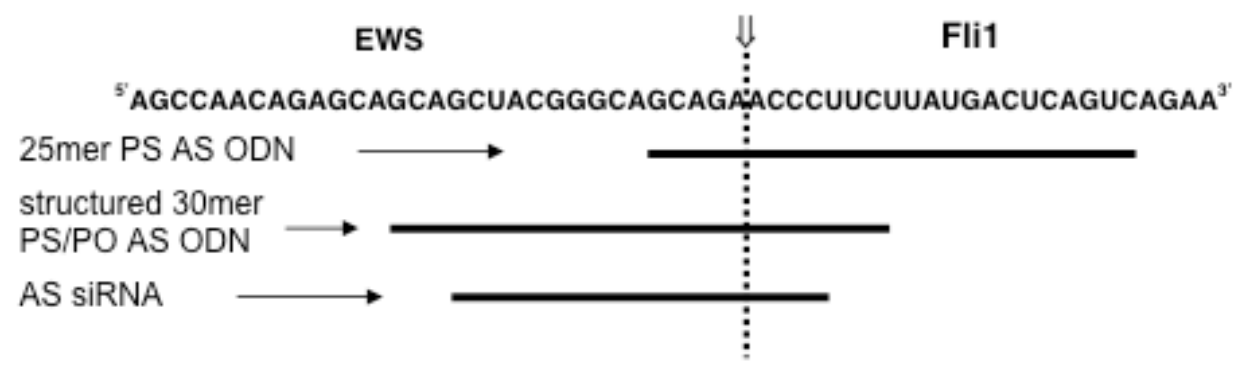

Fig. (1). Type 1 fusion point of EWS-Fli1 mRNA and the target sequences of 25mer PS AS ODN, structured 30mer PS/PO AS ODN and AS siRNA. 
Fli1, they have 12 common bases. The 25 mer is much more targeted to the Fli1 part of the fusion gene sequence whereas the $30 \mathrm{mer}$ is much more targeted to the EWS part. We also used an AS ODN fully constituted by phosphodiester linkages, which has the same sequence than the structured $30 \mathrm{mer}$ PS/PO AS-ODN but contains methyl modification at the 2' position of riboses present in the loop structure (structured 30mer 2'OMe PO AS ODN). The following ODNs were utilised as controls (CTL): ${ }^{\prime}-G^{*} C^{*} A^{*} G^{*} A * A * C^{*} C^{*} C^{*} T^{*}$ $\mathrm{T}^{*} \mathrm{C}^{*} \mathrm{~T}^{*} \mathrm{~T} * \mathrm{~A}^{*} \mathrm{~T} * \mathrm{G}^{*} \mathrm{~A} * \mathrm{C}^{*} \mathrm{~T}^{*} \mathrm{C}^{*} \mathrm{~A} * \mathrm{G}^{*} \mathrm{~T}^{*} \mathrm{C}^{*}-3$, (25mer PS CTL ODN), and 5'-CTCACCTTACTT*A*T*C*A*C*A*T* CATCTCCTCAT-3' (30mer PS/PO CTL ODN).

The sequences of the siRNA directed against the oncogene, Fig. (1), are as follows: 5'-GGGUUCUGCUGCCCG UAGC-d(UG)-3' (antisense strand AS siRNA), 5'-GCUACG GGCAGCAGAACCC-d(UU)-3' (sense strand AS siRNA) [9]. The control siRNA contains four mismatched bases (un-

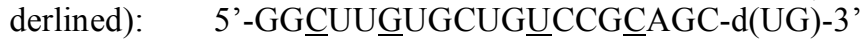
(antisense strand CTL siRNA), 5'-GCUGCGGACAGCA

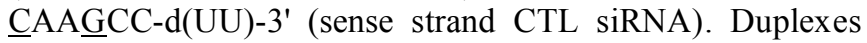
formation was performed by heating equimolar quantities of each strand for 2 minutes at $90{ }^{\circ} \mathrm{C}$, then 1 -hour incubation at $37^{\circ} \mathrm{C}$ in $100 \mathrm{mM}$ HEPES pH 7.4, $100 \mathrm{mM} \mathrm{NaCl}$ buffer.

\section{Transfection Conditions}

One day after seeding in 24-well or 6-well plates, EWSFli1 NIH/3T3 or NIH/3T3 cells were transfected with a mixture of ODN $(200 \mathrm{nM})$ or siRNA $(50 \mathrm{nM})$ and Cytofectin ${ }^{\mathrm{TM}}$ (ratio 1:2 w:w; GTS; San Diego, USA). Nucleic acid and transfection agent were both diluted in polystyrene tubes (Receptor Technologies; Oxon, England) containing $10 \mathrm{mM}$ HEPES pH 7.4, $100 \mathrm{mM} \mathrm{NaCl}$ buffer. The transfection volume was 1:10 of the final cell culture volume. The transfection mix was incubated 15 minutes at ambient temperature to make sure that the complexes were well established before adding to the cell culture medium. The cells were then incubated for 4 hours before analysis or the medium was replaced by a fresh one for a further incubation.

\section{Quantification of EWS-Fli1 and EWS mRNA Expres- sions by Real Time Q-PCR}

Cultured cells were transfected in 6-wells plates. After 4 hours treatment, the cells medium was discarded and $200 \mu \mathrm{L}$ of lysis buffer (4 M guanidium thiocyanate, $25 \mathrm{mM} \mathrm{Na}$ citrate $\mathrm{pH} 7,0.5 \%$ sarcosyl and $0.1 \mathrm{M} \beta$-mercaptoethanol) were added into each well. The lysate of 3 wells was pooled and total RNA was phenol extracted as follows. $40 \mu \mathrm{L}$ of $2 \mathrm{M} \mathrm{Na}$ acetate $\mathrm{pH} 4,400 \mu \mathrm{L}$ of water saturated phenol and $120 \mu \mathrm{L}$ of chlorophorm:isoamyl alcohol (49:1, v:v) were added. The mixture was centrifugated at $13000 \mathrm{rpm}$ for 15 minutes. 200 $\mu \mathrm{L}$ of isopropanol were mixed with $200 \mu \mathrm{L}$ of the supernatant and incubated at $-20{ }^{\circ} \mathrm{C}$ for 1 hour to salt out total RNA. Then, total RNA was precipitated after centrifugation at $13000 \mathrm{rpm}, 4{ }^{\circ} \mathrm{C}$, for 15 minutes. The pellet was washed with cold $75 \%$ ethanol and centrifugated once more, at $13000 \mathrm{rpm}, 4{ }^{\circ} \mathrm{C}$, for 5 minutes. After drying, the pellet was reconstituted in $10 \mu \mathrm{L}$ of distilled water which contained RNasin (1 U/ $\mu \mathrm{L}$; Promega, Mannheim, Allemagne). Concentration of RNA preparation was evaluated by $\mathrm{OD}_{260 \mathrm{~nm}}$ absorption and for quality by agarose gel electrophoresis followed by ethidium bromide staining. The cDNA was synthesized from $2 \mu \mathrm{g}$ total RNA in a $20 \mu \mathrm{L}$ reaction volume using
oligo(dT) (Promega) and reverse transcriptase M-MLV (Moloney Murine Leukemia Virus, Promega) for 1 hour at $42{ }^{\circ} \mathrm{C}$ according to the manufacturer's instructions.

Then, the expressions of the EWS gene and the EWSFlil oncogene were measured by real-time quantitative PCR using SYBR ${ }^{\circledR}$ Green I Dye (Applied Biosystems; Foster City, USA). Two sets of primers were used to study both EWS (forward 5'-AGCAGTTACTCTCAGCAGAACACC3', backward 5'-TCCACCAGGCTTATTGAAGCCACC-3') and EWS/Fli1 (forward 5'-AGCAGTTACTCTCAGCAGA ACACC-3', backward 5'-CCAGGATCTGATACGGATCT GGCTG-3') gene expression. PCR amplifications were performed according to Gene Amp ${ }^{\circledR}$ 5700, RNA Sybr ${ }^{\circledR}$ Green PCR protocol (Applied Biosystems) with 0.17 pmol and 0.34 pmol of backward and forward primers, respectively and a serial dilution of standard cDNA (0.01-100 nmol) in a $25 \mu 1$ final reaction volume. Thermocycling was conducted using ABI System Prism ${ }^{\circledR} 7000$ Sequence Detection System initiated by 15 minutes incubation at $94^{\circ} \mathrm{C}$ and followed by 40 cycles $\left(95^{\circ} \mathrm{C}, 15\right.$ seconds; $60^{\circ} \mathrm{C}, 60$ seconds) with a single fluorescent reading taken at the end of each cycle. $\mathrm{Ct}$ values were determined by the ABI Prism ${ }^{\circledR} 7000$ Sequence Detection System software. Standard curves were made to quantify the level of gene expression. PCR amplification was run for samples in duplicate and the representative results were expressed as the relative expression quantities of targets compared to those of untreated control that were normalised with the internal control $18 \mathrm{~s}$.

\section{Proliferation Assay}

After 24 hours treatment with antisense agent in 24-wells dishes, cellular proliferation was determined by MTT assay which measures the mitochondrial deshydrogenase succinate activity. After the medium replacement, a $0.5 \mathrm{mg} / \mathrm{mL}$ MTT (Sigma) solution was added, and the cells were incubated at $37^{\circ} \mathrm{C}$ for 2 hours. The cells were lysed overnight at $37{ }^{\circ} \mathrm{C}$ with the solution of $5 \% \mathrm{SDS}$ in $5 \mathrm{mM} \mathrm{HCl}$. Then, the OD of the supernatant was measured at the $570 \mathrm{~nm} / 630 \mathrm{~nm}$ wavelengths ratio with an automatic MRX II plate reader (Dynex Technologies; Chantilly, USA).

\section{Western-Blot Analysis}

After 24 hours treatment with structured 30mer PS/PO AS ODN in 6-wells dishes, ews-flil and ews protein expressions were measured by western-blot analysis according to standard procedures. Briefly, proteins were harvested in 100 $\mu 1$ lysis buffer (RIPA solution) containing Tris $50 \mathrm{mM}, \mathrm{NaCl}$ $150 \mathrm{mM}$, EDTA $1 \mathrm{mM}$ glycerol 10\%, NP 40 0.5\% and complete protease inhibitor (Roche, Germany) at $0{ }^{\circ} \mathrm{C}$ for 10 min. Then cell lysate were clarified by centrifugation for 10 $\min$ at $12000 \mathrm{~g}$ and conserved at $-80{ }^{\circ} \mathrm{C}$. Proteins concentrations were determined using BCA protein assay (Pierce). Proteins were separated on 4-10\% PAGE (Invitrogen) and transferred on BAF-83 nitro cellulose (Schleicher \& Schuell). Proteins were detected with specific anti EWS antibody (generous gift from Dr U. Kovar, Austria). Protein detection was performed by chemioluminescence (ECL, Amersham).

\section{Fluorescent Staining}

Cells were seeded onto glass slides and maintained for 24 hours. Then, a transfection was performed daily for 3 days 
(like previously described). $24 \mathrm{~h}$ latter, cells were fixed for 20 minutes at $4{ }^{\circ} \mathrm{C}$ with PBS containing $4 \%$ formaldehyde. The formaldehyde solution was neutralised with $50 \mathrm{mM} \mathrm{NH}_{4} \mathrm{Cl}$. Disruption of cell membranes cells was carried out for 5 minutes with $0.4 \%$ Triton X-100 in PBS. The cells were incubated for 30 minutes with RNase A $(10 \mu \mathrm{g} / \mathrm{mL}$ in PBS), and then for 20 minutes, at room temperature with $600 \mathrm{nM}$ DAPI (Sigma) and $1 \mu \mathrm{M}$ phalloidin-FITC (Sigma). Glass slides were mounted under Mowiol solution (Calbiochem; San Diego, USA) and observed through a fluorescence microscope (Zeiss; Le Pecq, France).

\section{Statistical Analysis}

For the proliferation assay, the percentage of cellular viability was calculated as the average of two independent experiments, and each one was made at least in triplicate (n $\geq 12$ ). GraphPad InStat software (San Diego, USA) was used to analyse the data. A two-tailed unpaired t-test was applied to compare the statistical significance of the differences between two groups. P values $<0.001$ were considered statistically extremely significant.

\section{RESULTS}

\section{Efficacy of the Antisense Agents: The EWS-Fli1 Onco- gene Expression}

We compared the different antisense agents, after a transfection of 4 hours, according to their ability to inhibit the expression of the human EWS-Flil oncogene which is expressed by transformed NIH/3T3 fibroblasts, Fig. (2). No specific variation in EWS-Fli1 oncogene expression was observed after the treatment of EWS-Fli1 NIH/3T3 cells by the 25mer PS AS ODN or its control sequence (63\% and $72 \%$ of untransfected cells, respectively). On the contrary, there was a significant difference between the structured 30mer PS/PO AS ODN and the 30mer PS/PO CTL ODN. The level of EWS-Fli1 mRNA expression was respectively reduced to $12 \%$ and $55 \%$ in comparison with the untreated EWS-Fli1 NIH/3T3 cells. To determine if the sequence of the structured ODN participates totally in the antisense effect or if the loop is mainly responsible, we replaced the RNase $\mathrm{H}$ sensitive phosphorothioate bases of the loop by RNase $\mathrm{H}$ resistant 2'-O-methyl modified oligodeoxynucleotides. The structured 30mer 2'OMe PO AS ODN inhibited the EWSFli1 mRNA expression as strongly as the unmodified ODN (13\% of EWS-Fli1 mRNA in untreated cells). The EWS-Fli1 mRNA expression was also specifically inhibited after transfection with AS siRNA (23\% of untransfected cells), whereas it was increased with CTL siRNA $(360 \%$ of untreated cells). We can notice that structured 30mer PS/PO AS ODN and AS siRNA were capable of silencing EWSFli1 gene expression to a similar level.

\section{Specificity of the Antisense Agents: The EWS Gene Ex- pression}

Even if the three studied antisense agents are all directed against the junction of the oncogene, their target sequences are quite different, Fig. (1). The 25mer PS AS ODN presents a stronger hybridisation to the Fli1 part of EWS-Fli1 whereas structured 30mer PS/PO AS ODN and AS siRNA present stronger hybridisation to the EWS part of EWS-Fli1. To measure the specificity of the antisense agents, we analysed the EWS gene expression after the transfection in

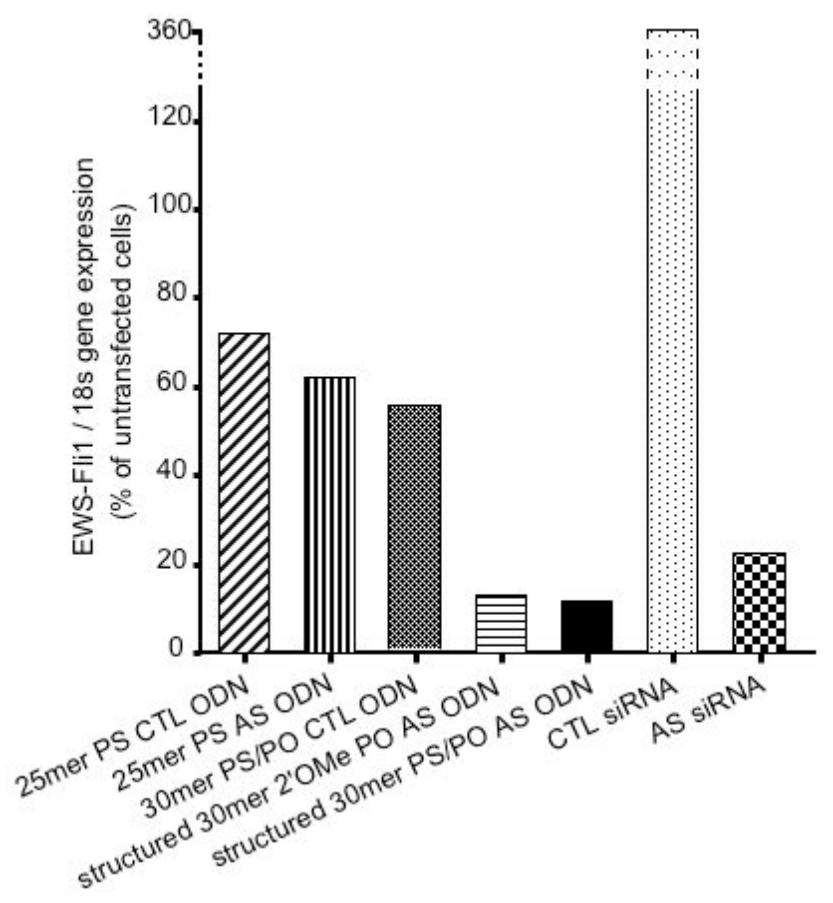

Fig. (2). Analysis of EWS-Fli1 mRNA expression levels by real time RT-PCR (Gene Amp ${ }^{\circledR} 5700$, RNA Sybr ${ }^{\circledR}$ Green PCR protocol, Applied Biosystems) after transfection of EWS-Fli1 NIH/3T3 cells with control ODNs, antisense ODNs, control siRNA or antisense siRNA for 4 hours.

The ODNs and siRNA are vectorised by Cytofectin ${ }^{\mathrm{TM}}$ at the concentrations of $200 \mathrm{nM}$ and $50 \mathrm{nM}$, respectively. Thermocycling was conducted using ABI System Prism ${ }^{\circledR} 7000$ Sequence Detection System initiated by 15 minutes incubation at $94^{\circ} \mathrm{C}$ and followed by 40 cycles $\left(95^{\circ} \mathrm{C}, 15\right.$ seconds; $60^{\circ} \mathrm{C}, 60$ seconds). Ct values were determined by the ABI Prism ${ }^{\circledR} 7000$ Sequence Detection System software. Levels of EWS-Fli1 mRNA expression are determined relatively to the level of $18 \mathrm{~s}$ mRNA expression and are expressed as the percentage of untransfected EWS-Fli1 NIH/3T3 cells. Each experiment is performed in triplicates.

NIH/3T3 and EWS-Fli1 NIH/3T3 for 4 hours, Fig. (3). The 25mer PS CTL ODN did not modify the EWS mRNA expression in the two cell lines (close to $100 \%$ in comparison with untreated cells). On the contrary, after transfection with 30mer PS/PO CTL ODN or CTL siRNA, the EWS mRNA level was two-fold increased in EWS-Fli1 NIH/3T3 (202\% and $204 \%$ of untransfected cells, respectively) and about 1.5 fold increased in NIH/3T3 (154\% and 167\% compared to untreated cells). With the 25mer PS AS ODN, we observed again a slightly stimulation of the EWS expression in EWSFli1 NIH/3T3 cells (142\% of the untransfected cells), whereas it was unmodified in NIH/3T3 (94\% in comparison with the untreated cells). In contrast, we noticed cell typedependent inhibitions of the EWS mRNA expression after transfection with structured 30mer PS/PO AS ODN or AS siRNA, Table 1. When EWS-Fli1 NIH/3T3 cells were transfected with the structured 30mer PS/PO AS ODN, the EWS gene expression was inhibited by $56 \%$ compared to untransfected cells. This inhibition was reduced to $7 \%$ in nontumour NIH/3T3 cells. In contrast, after transfection with AS siRNA, the reduction in EWS mRNA expression was higher 


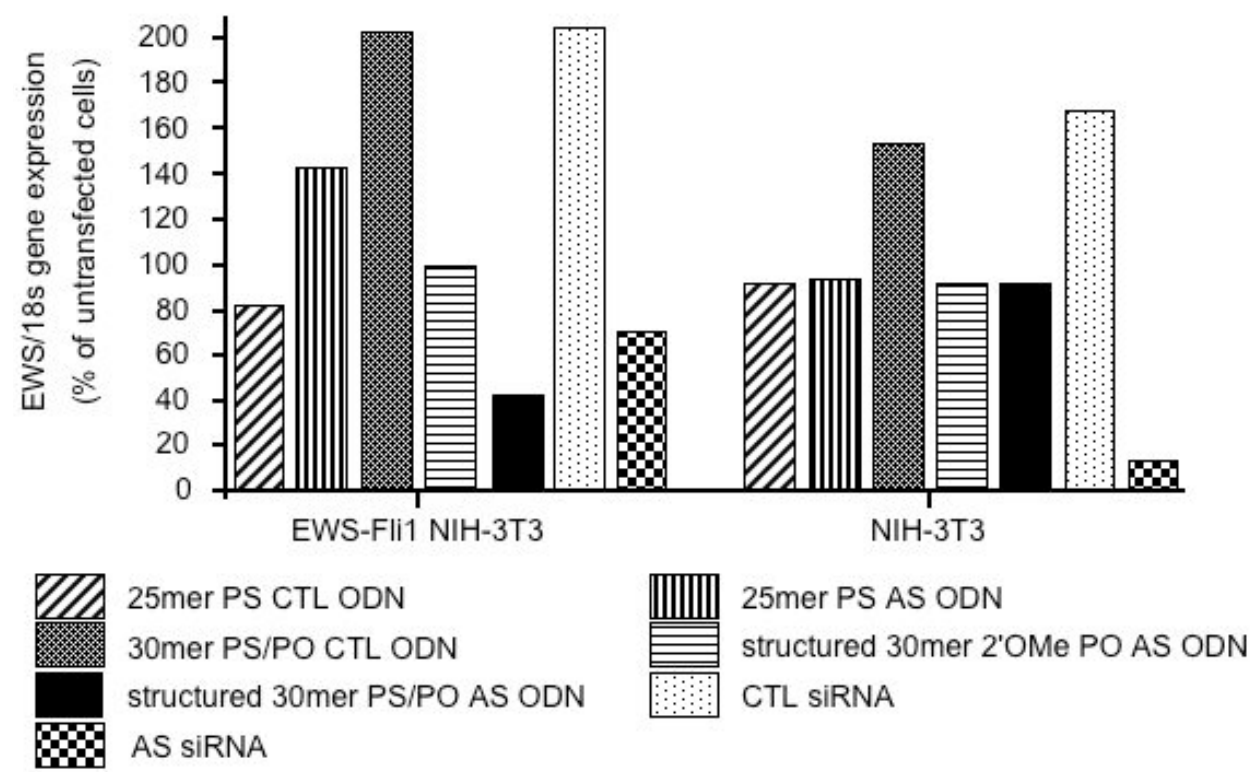

Fig. (3). Analysis of EWS mRNA expression levels by real-time RT-PCR (Gene Amp ${ }^{\circledR}$ 5700, RNA Sybr ${ }^{\circledR}$ Green PCR protocol, Applied Biosystems) after transfection of EWS-Fli1 NIH/3T3 or NIH/3T3 cells with control ODNs, antisense ODNs, control siRNA or antisense siRNA for 4 hours.

The ODNs and siRNA are vectorised by Cytofectin ${ }^{\mathrm{TM}}$ at the concentrations of $200 \mathrm{nM}$ and $50 \mathrm{nM}$, respectively. Thermocycling was conducted using ABI System Prism ${ }^{\circledR} 7000$ Sequence Detection System initiated by 15 minutes incubation at $94^{\circ} \mathrm{C}$ and followed by 40 cycles $\left(95^{\circ} \mathrm{C}, 15\right.$ seconds; $60^{\circ} \mathrm{C}, 60$ seconds). Ct values were determined by the ABI Prism ${ }^{\circledR} 7000$ Sequence Detection System software. Levels of EWS mRNA expression are determined relatively to the level of 18s mRNA expression and are expressed as the percentage of the respective untransfected EWS-Fli1 NIH/3T3 or NIH/3T3 cells. Each experiment is performed in triplicates.

Table 1. Comparison of EWS mRNA Expression Inhibition in NIH/3T3 Fibroblasts and EWS-Fli1 NIH/3T3 Cells, after Transfection for 4 Hours with the Structured 30mer PS/PO AS ODN (200nM) or the AS siRNA (50nM) both Vectorised by Cytofectin $^{\text {TM }}$

\begin{tabular}{|c|c|c|}
\hline \multirow{2}{*}{} & \multicolumn{2}{|c|}{ Inhibition of EWS gene expression in } \\
\cline { 2 - 3 } & $\begin{array}{c}\text { EWS-Fli1 NIH/3T3 cells } \\
\text { (\% of EWS-Fli1 NIH/3T3 untransfected cells) }\end{array}$ & $\begin{array}{c}\text { NIH/3T3 cells } \\
\text { (\% of NIH/3T3 untransfected cells) }\end{array}$ \\
\hline \hline structured 30mer PS/PO AS ODN & 56 & 7 \\
\hline AS siRNA & 28 & 85 \\
\hline
\end{tabular}

The expression of EWS mRNA is analysed by real time RT-PCR. It is determined relative to the expression of $18 \mathrm{~s}$ and is expressed as the percentage of untransfected cells NIH/3T3 or EWS-Fli1 NIH/3T3 cells. Each experiment is performed in triplicates.

in non-tumour NIH/3T3 cells (85\% of untransfected cells) than in tumour EWS-Fli1 NIH/3T3 cells (28\% of untransfected cells).

\section{Proliferation Assay}

To determine the cellular proliferation, we proceeded to a MTT assay 24 hours after transfecting NIH/3T3 or EWSFli1 NIH/3T3 cells, with the oligonucleotides, Fig. (4). There was no sequence-specific effect on the cellular growth neither with the 25mer PS AS ODN nor with the AS siRNA, since no difference was noted between the tumour and the parental cells after transfecting with the antisense agent or its respective control. On the contrary, after a transfection with the structured 30mer PS/PO AS ODN, the proliferation of EWS-Fli1 NIH/3T3 cells was significantly inhibited until $48 \%$ compared to the untransfected cells whereas the $30 \mathrm{mer}$ PS/PO CTL ODN had no significant effect on the cellular growth (only 10\% inhibition of EWS-Fli1 NIH/3T3 growth).
This specific inhibition was not observed in NIH/3T3 cells since the proliferation of NIH/3T3 after transfection with structured 30mer PS/PO AS and 30mer PS/PO CTL ODN were $88 \%$ and $87 \%$ of untransfected cells, respectively.

\section{ews-fli1 and ews Proteins Expression after Structured 30mer PS/PO AS ODN Treatment}

24 hours after transfecting EWS-Fli1 NIH/3T3 cells with the structured 30mer PS/PO AS ODN, we controled the ewsflil and ews proteins expression by western-blot analysis. We obtained no variation of the ews expression but a $38 \%$ inhibition of the ews-flil protein expression in comparison with the untransfected cells, Fig. (5).

\section{Reorganization of Actin Fibres}

To explore possible correlations between the inhibition of the EWS-Fli1 oncogene expression and cytoskeleton reorganization, we performed actin filament staining with phal- 


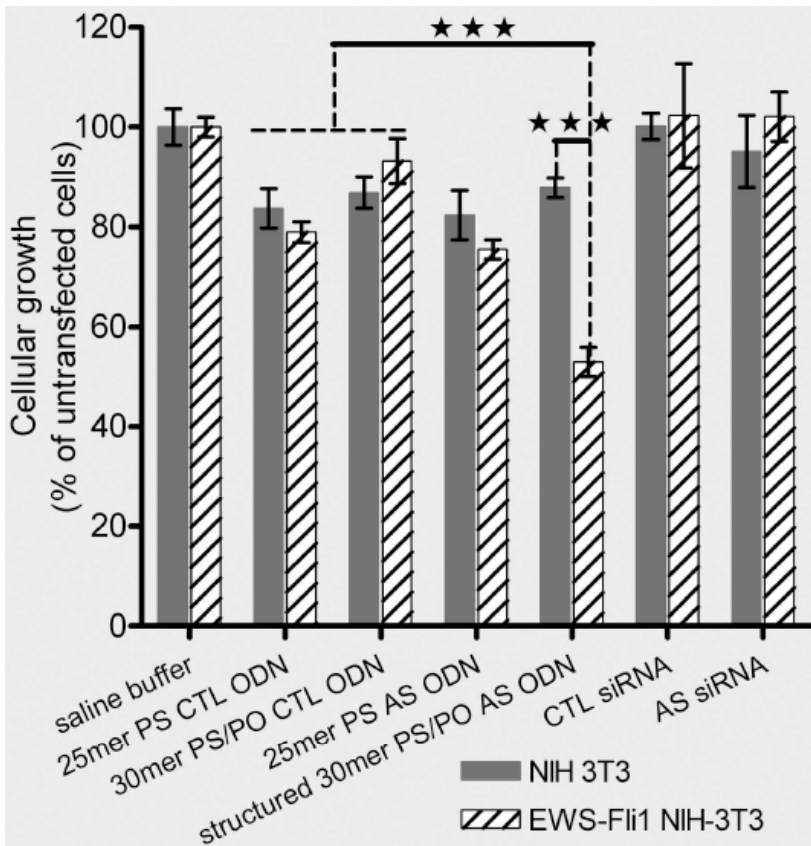

Fig. (4). Growth of NIH/3T3 and EWS-Fli1 cells in culture after 24 hours incubation with control ODNs, antisense ODNs, control siRNA or antisense siRNA.

The ODNs and siRNA are vectorised by Cytofectin ${ }^{\mathrm{TM}}$ at the concentrations of $200 \mathrm{nM}$ and $50 \mathrm{nM}$, respectively. Cellular viability is determined by MTT assay and expressed as the percentage of untransfected EWS-Fli1 NIH/3T3 cells. It is calculated as the average of two independent experiments that each one was made at least in triplicate $(n \geq 12)$.

loidin-FITC on NIH/3T3 and EWS-Fli1 NIH/3T3 fibroblasts before and after three successive transfections with various ODNs or siRNAs. Parental NIH/3T3 fibroblasts exhibit a highly organised actin filament network comprised of numerous, thick fibres. On the contrary, the actin cytoskeleton

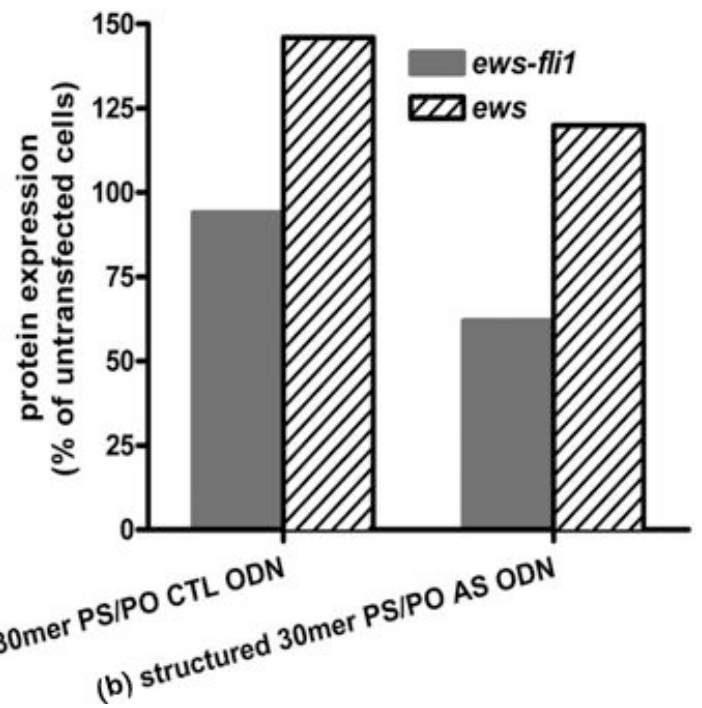

is entirely disrupted in EWS-Fli1 NIH/3T3 cells, showing no distinct fibres at all; and actin remains diffusely distributed within the cytoplasm, Fig. (6a). After transfecting tumour cells with 25 mer PS AS or its control and with structured 30mer PS/PO AS or its control, the same diffuse staining was observed inside the cells, Fig. (6b and $\mathbf{6 c}$ ). In contrast, the transfection of EWS-Fli1 NIH/3T3 cells with AS siRNA involved a less distributed staining all over the cytoplasm and actin fibres formation, whereas the actin cytoskeleton remained totally disrupted with CTL siRNA, Fig. (6d). It appears that treating tumour cells with AS siRNA resulted in morphological reversion: the actin cytoskeleton reorganization.

\section{DISCUSSION}

In this study, we compare three antisense agents which have been shown in several in vitro or in vivo experimental models to present some interesting potentialities to treat Ewing sarcoma $[6,9,11]$. To select the best agent among $25 \mathrm{mer}$ PS AS ODN, structured 30mer PS/PO AS ODN or AS siRNA, we used fibroblastic NIH/3T3 cell line which had been stably transfected with the human EWS-Fli1 oncogene. The advantage of this in vitro model of Ewing sarcoma is its own negative control: the non-tumour parental NIH/3T3 fibroblasts. The antisense agents were vectorised with cationic lipids. The concentrations of $200 \mathrm{nM}$ and $50 \mathrm{nM}$ were used for ODNs and siARNs, respectively. These concentrations were optimised to obtain similar levels of oncogene expression inhibition. Moreover this inhibition is the highest one with the lowest toxicity. The effects of these three antisense agents were studied according to three parameters: (a) the fluctuation of the oncogene and the EWS gene expressions, not only in tumour cells but also in parental NIH/3T3 fibroblasts, (b) the modification of tumour and non-tumour cell proliferation and finally (c) the reorganization of actin cytoskeleton.

We observed the stimulation of both the oncogene (after transfection with the CTL siRNA) and the EWS gene ex-

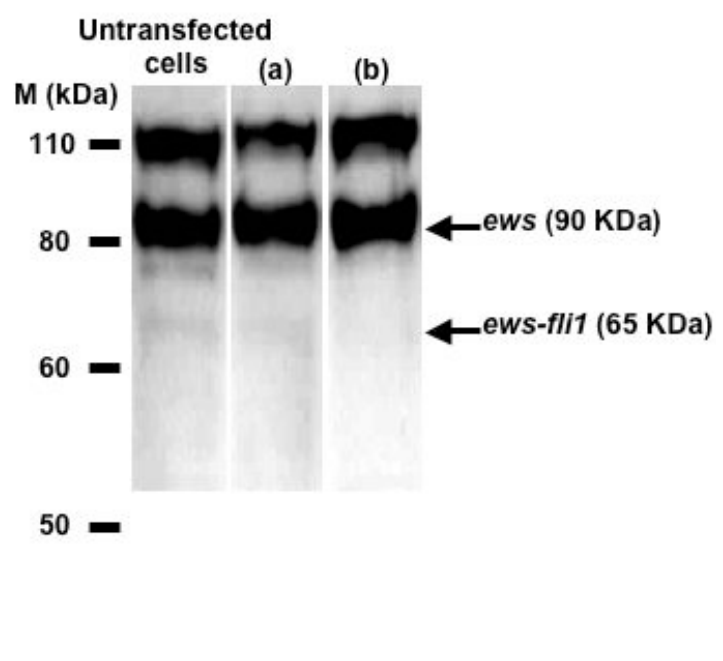

Fig. (5). Analysis of ews-fli1 and ews proteins expression by western-blot after transfection of EWS-Fli1 NIH/3T3 cells with (a) 30mer PS/PO CTL ODN or (b) structured 30mer PS/PO AS ODN for 24 hours.

The ODNs - at the concentration of $200 \mathrm{nM}$ - are vectorized by cytofectin ${ }^{\mathrm{TM}}$. Levels of proteins expression are determined relatively to an irrelevant $38 \mathrm{kDa}$ protein and are expressed as the percentage of untransfected EWS-Fli1 NIH/3T3 cells. Representative experiment. 

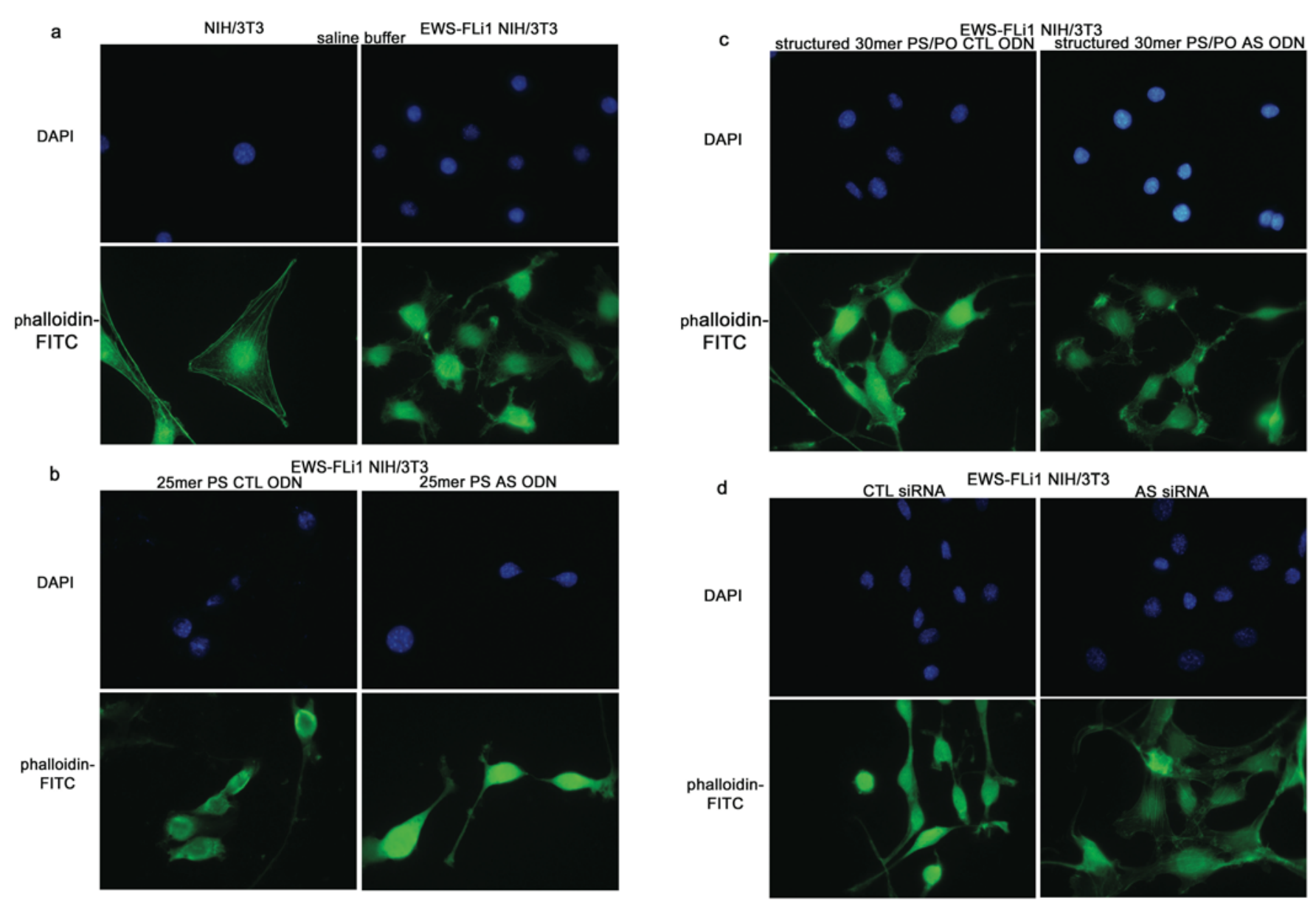

Fig. (6). Architecture of the actin cytoskeleton architecture in EWS-Fli1 NIH/3T3 cells after three daily consecutive transfections with antisense ODNs, or siRNA bound to Cytofectin ${ }^{\mathrm{TM}}$.

The samples are analysed by fluorescence microscopy after the staining of actin fibres by phalloidin-FITC. (a) Untransfected NIH/3T3 and EWS-Fli1 NIH/3T3 cells. (b) EWS-Fli1 NIH/3T3 transfected with 200nM of 25mer PS CTL or AS ODN. (c) EWS-Fli1 NIH/3T3 transfected with $200 \mathrm{nM}$ of $30 \mathrm{mer}$ PS/PO CTL or structured 30mer PS/PO AS ODN. (d) EWS-Fli1 NIH/3T3 transfected with 50nM of 25mer CTL or AS siRNA.

pression (after transfection with the 30mer PS/PO CTL ODN, the 25mer PS AS ODN, or the CTL siRNA). The variation in expression of untargeted genes is unfortunately often described [22-25].

According to our findings, the 25 mer PS AS ODN does not specifically inhibit the oncogene expression after 4 hours in comparison with the ODN CTL. Moreover, after 4 hours, the EWS gene expression was a 1.5-fold increased in tumour EWS-Fli1 cells compared to untransfected cells but not modified in parental NIH/3T3 fibroblasts (94\% of untreated cells). The cellular growth inhibition was unspecific (both in term of cell type and ODN sequence). In our experimental conditions, the antisense activity of 25 mer PS AS ODN may not be showed because of an unspecific intracellular penetration and traffic.

Like previous studies [14], we confirm that the structured 30mer PS/PO AS ODN induced strong and almost specific inhibitions of EWS-Fli1 mRNA expression (measured by real-time Q-PCR) and cellular proliferation in tumour EWSFli1 NIH/3T3 cells ( $88 \%$ and $48 \%$ in comparison with untransfected cells, respectively). The 2'-O-methyl modified structured 30mer PO AS ODN inhibited the EWS-Fli1
mRNA expression as strongly as the unmodified ODN $(87 \%)$. Therefore, the alkyl modification in the loop domain does not disrupt the antisense activity of the antisense agent. As 2'-O-methylation modification blocks RNase $\mathrm{H}$ cleavage of the target mRNA, we can assert that the loop is not mainly responsible of the inhibition of the oncogene expression. In consequence, to carry on its antisense effect, the structured 30mer PS/PO AS needs to be disorganised and to become linear.

The penetration of ODNs doesn't differ between the two cellular types (data not shown). Therefore, it is difficult to explain why EWS mRNA expression was more down regulated by the structured 30mer PS/PO AS ODN in tumour cells than in NIH/3T3 (respectively $44 \%$ and $93 \%$ of EWS mRNA level in untransfected cells). By contrast, the structured 30mer 2'OMe PO AS ODN did not modify the expression of EWS both in tumour and NIH/3T3 cells (respectively $93 \%$ and $101 \%$ of untransfected cells). Indeed, the 2'-Oalkyl modifications only concern bases directed against the EWS part of the junction point. Since they are known to inhibit the RNase $\mathrm{H}$ cleavage, the area of RNase $\mathrm{H}$ cut in the EWS part is reduced in comparison with the unmodified AS ODN. Then, the EWS gene is not down regulated [26-29]. 
The consequences of inhibited EWS gene expression in nontumour cells are still unknown. For therapeutic purposes, it would be preferable to maintain EWS mRNA expression at its physiological level. So, finding almost no variation of EWS gene expression in normal NIH/3T3 cells after transfection with the structured 30mer PS/PO AS ODN is very valuable for its promising use in clinical trials.

In our in vitro Ewing sarcoma model, our results point out that the AS siRNA has two targets: not only the EWSFli1 oncogene but also the EWS gene. Indeed, in the tumour EWS-Fli1 NIH/3T3 cells where these two targets are present, the molecules of AS siRNA are distributed according to their affinity and the availability of the two targets (28\% and $77 \%$ inhibitions of EWS and EWS-Fli1 expressions compared to untreated cells, respectively). But, in the nontumour NIH/3T3 fibroblasts, all the molecules of AS siRNA are free to inhibit just the remaining target, the EWS gene (inhibition of EWS expression at 85\% compared to untransfected cells). It is important to notice that the sequence of the AS siRNA has more homology with the EWS part of EWSFli1 (15 nucleotides out of 19) than the Fli1 part. It is well known that siRNAs can tolerate one to several mismatches to the mRNA targets and that only a partial homology is sufficient for an effective silencing of untargeted protein [23]. On the contrary, with the same siRNA, Dohjima et al. found only $5 \%$ inhibition of EWS [9]. But the siRNA was intracellularly expressed and the housekeeping gene chosen to normalize the inhibitions was not the same. In a therapeutic point of view, the ability of siRNA to strongly inhibit EWS expression could represent a serious problem unless tumour cells specific transfection is guaranteed.

No inhibition of tumour cell proliferation occurred after AS siRNA treatment (cellular growth of $98.6 \%$ in comparison with the untreated cells). This is a very unexpected result if we consider that oncogene expression and cell growth inhibitions are reliable. Indeed, as previously illustrated in another Ewing sarcoma model [9], we obtained $77 \%$ inhibition of oncogene mRNA expression after the transfection of EWS-Fli1 NIH/3T3 cells with AS siRNA. This down regulation was only $11 \%$ lower than the one mediated by structured 30 mer PS/PO AS ODN with which the cellular proliferation is $48 \%$ inhibited. This difference seems too small to justify that no consequence with the AS siRNA was observed at the cellular proliferation level. On the contrary, in the same tumour cells, the inhibition of EWS mRNA expression mediated by AS siRNA was twice lower than the one induced by the structured 30mer PS/PO AS ODN (28\% and 56\% compared with untransfected cells, respectively). Spahn et al. showed that homo- and hetero-oligomerisation of ews and ews-flil proteins occurs [30]. However, their functional consequences remain to be elucidated. Accordingly, we first supposed that in tumour EWS-Fli1 NIH/3T3, the simultaneous downregulation of EWS-Fli1 and EWS by the structured 30mer PS/PO AS ODN might have a cooperative effect via consecutive down regulation at protein level. This hypothesis could explain why the inhibition of tumour cells proliferation only happened after the transfection with structured 30mer PS/PO AS ODN and not with AS siRNA. Several authors reported controversial conclusions regarding genesis of Ewing sarcoma family of tumors. Kovar et al. have shown that ews protein is dispensable for Ewing tumor growth. Some authors suppose that the heterodimerisation of ews-flil with ews proteins may be involved in the DNA-binding independent pathway of the Ewing's tumours genesis [30-33]. So, we proceeded to the western-blot analysis 24 hours after transfecting EWS-Fli1 NIH/3T3 cells with structured 30mer PS/PO AS ODN. Consistent with the Q-PCR results, we detected $38 \%$ inhibition of ews-flil protein expression compared to untransfected cells. This inhibition is quite similar to which was obtained in the same conditions by Maksimenko et al. [14]. Unfortunately, no decrease of ews protein expression was quantified. We concluded that the effect observed on cellular proliferation after AS treatment is not due to the simultaneous down regulation of ews-flil and ews. No evidence was found about the role of the heterodimerisation of ews-flil with ews in the Ewing sarcoma pathogenesis. We had to notice that there is a correlation between RNA and protein down regulations for EWS-FLI1 but not for EWS. To explain this, we can suppose that ews-flil and ews proteins are differently regulated. The ews-flil turnover may be more rapid than ews one.

Finally, to explain why the structured 30mer PS/PO AS ODN inhibited the cellular growth and siRNA did not, while both down regulated the oncogene mRNA expression, we may find an explanation with microRNA (miRNA) and offtarget effect concepts. Indeed, it is now well known that transcripts needn't total complementarity with a siRNA to be targeted for knockdown by the RNAi pathway [23, 34-38]. Bioinformatic tools are more and more available on the web. Thanks to the miRBase database [39], we identified sequence homologies between AS siRNA and two miRNAs: mmu-mir-883a-5p and mmu-mir-346 (respectively ten and seven bases in common). Even if it doesn't prove that the AS siRNA acts as a miRNA, doubts remain. And caution is especially necessary since mmu-mir- $883 \mathrm{a}-5 \mathrm{p}$ and mmu-mir346 possess a large number of target mRNA [39, 40]. The most annoying is the discovery of 14 murine genes of which the sequences partially match with the sense or the antisense AS siRNA strand [41, 42]. Among 19 bases of the siRNA strands, the number of mismatches varies from 2 to 4 . But all present contiguous match part between 9 to 15 bases which might be enough for RNAi process. Therefore, it seems that the AS siRNA could possess a lot of off-target genes. One or several of them like Src (see [43-46] for reviews) could act in cell growth. And, we hypothesize that we cannot observe a cellular proliferation effect mediated by AS siRNA because of those off-target genes that remain to be identified.

At last, cell transformation often involves changes in cell architecture, essentially linked to profound cytoskeleton rearrangements. In particular, in our in vitro model of Ewing sarcoma, the expression of EWS-Fli1 in NIH/3T3 fibroblasts induces the complete disruption of the actin fibres network [20,21]. The reorganization of actin fibres cytoskeleton was only obtained after transfection with AS siRNA. This illustrates completely what Bertrand et al. showed: the effects of siRNAs are lasting for a longer time than the ones of AS ODN in cell culture [22]. In this way, we expect that structured 30mer AS ODN could also involve this phenotypic reversion if it is maintained intact for a longer time.

\section{CONCLUSION}

To conclude, we observe that according to its efficacy, its specificity and the absence of induced cell phenotypic rever- 
sion, the 25mer PS AS ODN does not present interesting enough features in our in vitro murine model of Ewing sarcoma. Our results confirm that structured 30mer PS/PO AS ODN and AS siRNA have both promising potentialities to design a new therapy directed against Ewing sarcoma. They highly inhibit EWS-Fli1 mRNA expression. AS siRNA causes an interesting effect on actin cytoskeleton reorganization. However we cannot exclude that it has also an action on off-target genes. To prevent undesired effects systems allowing either a direct expression of a shRNA in tumor cells or a tumor targeted vectorisation of siRNA should be developed. With a strong effect on cellular proliferation and no serious adverse effect detected, structured 30mer PS/PO AS ODN appears to be the best of the three tested antisense agents. It is efficient but needs a longer half-life through the improvement of its resistance to nuclease degradation. It is now necessary to design the best partner to protect and vectorise it inside the cells.

\section{ACKNOWLEDGEMENTS}

The authors thank Mr. Andréi Maksimenko for his scientific advises. We express gratitude to Dr. Frank André and Dr. Alan Gewirtz for their very helpful reading and comments. This work was partly realised at the functional genomics unit at IGR, Villejuif. The authors acknowledge the financial support of the Association pour la Recherche sur le Cancer (ARC) and the EU Commission through the project PROTHETS.

\section{REFERENCES}

[1] May WA, Lessnick SL, Braun BS, et al. The Ewing's sarcoma EWS/FLI-1 fusion gene encodes a more potent transcriptional activator and is a more powerful transforming gene than FLI-1. Mol Cell Biol 1993; 13: 7393-8.

[2] May WA, Gishizky ML, Lessnick SL, et al. Ewing sarcoma 11;22 translocation produces a chimeric transcription factor that requires the DNA-binding domain encoded by FLI1 for transformation. Proc Natl Acad Sci USA 1993; 90: 5752-6.

[3] Khoury JD. Ewing sarcoma family of tumors. Adv Anat Pathol 2005; 12: 212-20.

[4] Ouchida M, Ohno T, Fujimura Y, Rao VN, Reddy ES. Loss of tumorigenicity of Ewing's sarcoma cells expressing antisense RNA to EWS-fusion transcripts. Oncogene 1995; 11: 1049-54.

[5] Kovar H, Aryee DN, Jug G, et al. EWS/FLI-1 antagonists induce growth inhibition of Ewing tumor cells in vitro. Cell Growth Differ 1996; 7: 429-37.

[6] Tanaka K, Iwakuma T, Harimaya K, Sato H, Iwamoto Y. EWSFli1 antisense oligodeoxynucleotide inhibits proliferation of human Ewing's sarcoma and primitive neuroectodermal tumor cells. J Clin Invest 1997; 99: 239-47.

[7] Lambert G, Bertrand JR, Fattal E, et al. EWS Fli-1 antisense nanocapsules inhibits Ewing sarcoma-related tumor in mice. Biochem Biophys Res Commun 2000; 279: 401-6.

[8] Matsumoto Y, Tanaka K, Nakatani F, Matsunobu T, Matsuda S, Iwamoto Y. Downregulation and forced expression of EWS-Fli1 fusion gene results in changes in the expression of $G(1)$ regulatory genes. Br J Cancer 2001; 84: 768-75.

[9] Dohjima T, Lee NS, Li H, Ohno T, Rossi JJ. Small interfering RNAs expressed from a Pol III promoter suppress the EWS/Fli-1 transcript in an Ewing sarcoma cell line. Mol Ther 2003; 7: 811-6.

[10] Maksimenko A, Malvy C, Lambert G, et al. Oligonucleotides targeted against a junction oncogene are made efficient by nanotechnologies. Pharm Res 2003; 20: 1565-7.

[11] Maksimenko A, Lambert G, Bertrand JR, Fattal E, Couvreur P, Malvy C. Therapeutic potentialities of EWS-Fli-1 mRNA-targeted vectorized antisense oligonucleotides. Ann NY Acad Sci 2003; 1002: 72-7.

[12] Nozawa S, Ohno T, Banno Y, et al. Inhibition of platelet-derived growth factor-induced cell growth signaling by a short interfering
RNA for EWS-Fli1 via down-regulation of phospholipase D2 in Ewing sarcoma cells. J Biol Chem 2005; 280: 27544-51.

[13] Maksimenko A, Malvy C. Oncogene-targeted antisense oligonucleotides for the treatment of Ewing sarcoma. Expert Opin Ther Targets 2005; 9: 825-30.

[14] Maksimenko A, Polard V, Villemeur M, et al. In vivo potentialities of EWS-Fli-1 Targeted antisense oligonucleotides-nanospheres complexes. Ann N Y Acad Sci 2005; 1058: 52-61.

[15] Matsunobu T, Tanaka K, Nakamura T, et al. The possible role of EWS-Fli1 in evasion of senescence in Ewing family tumors. Cancer Res 2006; 66: 803-11.

[16] Toub N, Bertrand JR, Tamaddon A, et al. Efficacy of siRNA nanocapsules targeted against the EWS-Flil oncogene in Ewing sarcoma. Pharm Res 2006; 23: 892-900.

[17] Bertolotti A, Melot T, Acker J, Vigneron M, Delattre O, Tora L. EWS, but not EWS-FLI-1, is associated with both TFIID and RNA polymerase II: interactions between two members of the TET family, EWS and hTAFII68, and subunits of TFIID and RNA polymerase II complexes. Mol Cell Biol 1998; 18: 1489-97.

[18] Araya N, Hirota K, Shimamoto Y, et al. Cooperative interaction of EWS with CREB-binding protein selectively activates hepatocyte nuclear factor 4-mediated transcription. J Biol Chem 2003; 278 : 5427-32.

[19] Janknecht R. EWS-ETS oncoproteins: the linchpins of Ewing tumors. Gene 2005; 363: 1-14.

[20] Pawlak G, Helfman DM. Cytoskeletal changes in cell transformation and tumorigenesis. Curr Opin Genet Dev 2001; 11: 41-7.

[21] Amsellem V, Kryszke MH, Hervy M, et al. The actin cytoskeletonassociated protein zyxin acts as a tumor suppressor in Ewing tumor cells. Exp Cell Res 2005; 304: 443-56.

[22] Bertrand JR, Pottier M, Vekris A, Opolon P, Maksimenko A, Malvy C. Comparison of antisense oligonucleotides and siRNAs in cell culture and in vivo. Biochem Biophys Res Commun 2002; 296: 1000-4.

[23] Jackson AL, Bartz SR, Schelter J, et al. Expression profiling reveals off-target gene regulation by RNAi. Nat Biotechnol 2003; 21 : 635-7.

[24] Persengiev SP, Zhu X, Green MR. Nonspecific, concentrationdependent stimulation and repression of mammalian gene expression by small interfering RNAs (siRNAs). RNA 2004; 10: 12-8.

[25] Bilanges B, Stokoe D. Direct comparison of the specificity of gene silencing using antisense oligonucleotides and RNAi. Biochem J 2005; 388: 573-83.

[26] Iribarren AM, Sproat BS, Neuner P, Sulston I, Ryder U, Lamond AI. 2'-O-alkyl oligoribonucleotides as antisense probes. Proc Natl Acad Sci USA 1990; 87: 7747-51.

[27] Gallo M, Montserrat JM, Iribarren AM. Design and applications of modified oligonucleotides. Braz J Med Biol Res 2003; 36: 143-51.

[28] Yoo BH, Bochkareva E, Bochkarev A, Mou TC, Gray DM. 2'-Omethyl-modified phosphorothioate antisense oligonucleotides have reduced non-specific effects in vitro. Nucleic Acids Res 2004; 32: 2008-16.

[29] Chen X, Dudgeon N, Shen L, Wang JH. Chemical modification of gene silencing oligonucleotides for drug discovery and development. Drug Discov Today 2005; 10: 587-93.

[30] Spahn L, Siligan C, Bachmaier R, Schmid JA, Aryee DN, Kovar H. Homotypic and heterotypic interactions of EWS, FLI1 and their oncogenic fusion protein. Oncogene 2003; 22: 6819-29.

[31] Kovar H, Jug G, Hattinger C, et al. The EWS protein is dispensable for Ewing tumor growth. Cancer Res 2001; 61: 5992-7.

[32] Jaishankar S, Zhang J, Roussel MF, Baker SJ. Transforming activity of EWS/FLI is not strictly dependent upon DNA-binding activity. Oncogene 1999; 18: 5592-7.

[33] Welford SM, Hebert SP, Deneen B, Arvand A, Denny CT. DNA binding domain-independent pathways are involved in EWS/FLI1mediated oncogenesis. J Biol Chem 2001; 276: 41977-84.

[34] Alema LM, Doench J, Sharp PA. Comparison of siRNA-induced off-target RNA and protein effects. DNA 2007; 13: 385-95.

[35] Saxena S, Jonsson ZO, Dutta A. Small RNAs with imperfect match to endogenous mRNA repress translation. Implications for offtarget activity of small inhibitory RNA in mammalian cells. J Biol Chem 2003; 278: 44312-9.

[36] Haley B, Zamore P. Kinetic analysis of the RNAi enzyme complex. Nat Struct Mol Biol 2004; 11: 559-606. 
[37] Birmingham A, Anderson EM, Reynolds A, et al. 3' UTR seed matches, but not overall identity, are associated with RNAi offtargets. Nat Methods 2006; 3: 199-204.

[38] Jackson AL, Burchard J, Schelter J, et al. Widespread siRNA “offtarget" transcript silencing mediated by seed region sequence complementarity. RNA 2006; 12: 1179-87.

[39] Wellcome Trust Genome Campus, Hinxton, Cambridge, CB10 1SA, UK. [Updated 2008 Sep 8]. Available from: http://microrna.sanger.ac.uk

[40] microRNA.org: a resource for predicted microRNA targets and expression. Memorial Sloan-Kettering Cancer Center. [Updated 2008 Sep 8]. Available from: http://www.microrna.org

[41] Specificity Server: An siRNA design program for determining siRNA specificity. Available from: http://informaticseskitis.griffith.edu.au/Specificity Server
[42] Chalk AM, Sonnhammer EL. siRNA specificity searching incorporating mismatch tolerance data. Bioinformatics 2008; 24 (10): 1316-7.

[43] Summy JM, Gallick GE. Src family kinases in tumor progression and metastasis. Cancer Metast Rev 2003; 22: 337-58.

[44] Parsons SJ, Parsons JT. Src family kinases, key regulators of signal transduction. Oncogene 2004; 23:7906-9.

[45] Tuhácková Z. Molecular therapeutics - Lessons from the role of Src in cellular signalling. Fol Biol (Praha) 2005; 51: 114-20.

[46] Johnson FM, Gallick GE. Src family nonreceptor tyrosine kinases as molecular targets for cancer therapy. Anticancer Agents Med Chem 2007; 7(6): 651-9.

Received: January 29, 2009

Revised: May 19, 2009

Accepted: May 28, 2009

(C) Villemeur et al.; Licensee Bentham Open.

This is an open access article licensed under the terms of the Creative Commons Attribution Non-Commercial License (http://creativecommons.org/licenses/by-nc/3.0/) which permits unrestricted, non-commercial use, distribution and reproduction in any medium, provided the work is properly cited. 